\title{
Reduced synaptic activity in neuronal networks derived from embryonic stem cells of murine Rett syndrome model
}

\author{
Lydia Barth, Rosmarie Sütterlin, Markus Nenniger and Kaspar E. Vogt*
}

Neurobiology and Pharmacology, Biozentrum, University of Basel, Basel, Switzerland

\section{Edited by:}

Andrea Barberis, Fondazione Istituto

Italiano di Tecnologia, Italy

\section{Reviewed by:}

Melanie A. Woodin, University of Toronto, Canada

Annalisa Scimemi, University at Albany - The State University of New York, USA

\section{*Correspondence:}

Kaspar E. Vogt, Neurobiology and Pharmacology, Biozentrum, University of Basel, Klingelbergstrasse 50/70, 4056 Basel, Switzerland e-mail:kaspar.vogt@unibas.ch
Neurodevelopmental diseases such as the Rett syndrome (RTT) have received renewed attention, since the mechanisms involved may underlie a broad range of neuropsychiatric disorders such as schizophrenia and autism. In vertebrates early stages in the functional development of neurons and neuronal networks are difficult to study. Embryonic stem cellderived neurons provide an easily accessible tool to investigate neuronal differentiation and early network formation. We used in vitro cultures of neurons derived from murine embryonic stem cells missing the methyl-CpG-binding protein 2 (MECP2) gene (MeCP2-/y) and from wild type cells of the corresponding background. Cultures were assessed using whole-cell patch-clamp electrophysiology and immunofluorescence. We studied the functional maturation of developing neurons and the activity of the synaptic connections they formed. Neurons exhibited minor differences in the developmental patterns for their intrinsic parameters, such as resting membrane potential and excitability; with the MeCP2-/y cells showing a slightly accelerated development, with shorter action potential half-widths at early stages. There was no difference in the early phase of synapse development, but as the cultures matured, significant deficits became apparent, particularly for inhibitory synaptic activity. MeCP2-/y embryonic stem cell-derived neuronal cultures show clear developmental deficits that match phenotypes observed in slice preparations and thus provide a compelling tool to further investigate the mechanisms behind RTT pathophysiology.

Keywords: Rett syndrome, stem cell-derived neurons, neurodevelopment, electrophysiology, excitability, synaptic activity

\section{INTRODUCTION}

Rett (1966) described an unusual neurodevelopmental disorder in girls, now called Rett syndrome (RTT). Today the key diagnostic criteria for RTT are stereotypic hand movements, deficits in motor coordination, speech disorders, and autistic behavior (Hagberg and Hagberg, 1997). Children develop normally for 6-18 months after birth, reaching the usual motor, language and social milestones. This brief period of developmental progress is followed by stagnation with growth arrest and microcephaly. During the following rapid regression phase, the previously acquired skills are lost and a variety of neurological symptoms develop. These include sleep disturbances, problems with gait, decelerated head growth, breathing arrhythmia, stereotypical hand movements, loss of motor coordination, and seizures (Zoghbi, 2003; Moretti and Zoghbi, 2006; Chahrour and Zoghbi, 2007).

Amir etal. (1999) identified the primary cause of RTT as a defect in the MECP2 gene on the $\mathrm{X}$ chromosome, coding for the methyl-CpG-protein 2 (MeCP2). More than 95\% of individuals with classic RTT carry de novo mutations. MeCP2 is highly enriched in neurons in the central nervous system (Zhou et al., 2006). It regulates genes essential for neuronal survival, dendritic growth, synaptogenesis, and synaptic plasticity (Fukuda et al., 2005; Chang et al., 2006; Smrt et al., 2007). The function of MeCP2-targeted genes seems especially important in GABAergic neurons (Huang et al., 2007; Chao et al., 2010).
GABAergic interneurons provide the main inhibitory function in the central nervous system and thereby contribute to the essential balance between excitation and inhibition. A disturbed excitation/inhibition balance will in severe cases result in epileptic discharges, which are found in $70-90 \%$ of RTT patients (Nissenkorn et al., 2010).

Generation of different mouse models in 2001 by targeting of the $M E C P 2$ gene has provided significant advances in understanding of MeCP2 function and mimicking relevant aspects of human RTT (Chen et al., 2001; Guy et al., 2001; Shahbazian et al., 2002). $\mathrm{MeCP} 2$ - null male mice (MeCP2-/y) were generated by replacing exons 3 and 4 of MECP2 starting in early embryonic development (Guy et al., 2001). Most studies use such male hemizygous mice because they develop a severe and characteristic behavioral phenotype much earlier than female heterozygous mice. The mice develop motor impairments, tremor, breathing abnormalities, limb stereotypies, and epilepsy as in the human condition (Colic et al., 2013). Remarkably, it was later shown that re-expression of endogenous MeCP2 can reverse aspects of RTT in the adult (Guy et al., 2007). Within a few weeks the affected mice were largely indistinguishable from their wild type (wt) controls. This does not yet suggest a prompt therapeutic approach to RTT but it clearly establishes the principle of reversibility in this mouse model.

The causal link between MeCP2 dysfunction and the neurobehavioral phenotype is still unclear. Given the reversibility of the 
phenotype, a better understanding of the neuronal phenotype becomes more and more important. Especially early developmental stages are hard to study functionally since the neurons are not easily accessible for targeted manipulations at these stages.

Murine embryonic stem cell (mES)-derived neurons allow a straightforward functional analysis of neuron maturation from very early stages up to network formation.

Here we investigate the differentiation of neural precursors derived from MeCP2-/y mice and from the corresponding E14Tg2a wt background. We compared intrinsic parameters, such as resting membrane potential (RMP), the function of voltagegated sodium- and potassium channels as well as generation of action potentials (APs) from immature and mature neurons. As soon as the neurons formed synaptic networks, we studied spontaneous excitatory and inhibitory synaptic activity and its maturation over time.

\section{METHODS AND MATERIALS CELL CULTURE AND DIFFERENTIATION}

Embryonic stem cells derived from a E14Tg2a background with the MeCP2+/y (wt) and MeCP2-/y genotype were cultured and differentiated into neurons as described (Bibel et al., 2007). Briefly, after 4 days of embryoid body formation these are treated with $5 \mu \mathrm{M}$ all-trans-retinoic acid (Sigma Inc., Buchs, Switzerland) for additional 4 days. Embryoid bodies are dissociated and neuronal precursors were plated on poly-L-ornithine (Sigma Inc., Buchs, Switzerland)/laminin (Roche Inc., Buchs, Switzerland) - coated glass cover slips (Assistent, Karl Hecht GmbH, Sondheim/Rhön, Germany). At day in vitro (DIV) 0 and 1 neuronal precursors were cultured in neural medium containing DMEM/F12, N-2 Supplement (100X) and penicillin/streptomycin and $1 \mathrm{mM}$ glutamine (all Invitrogen Inc., Lucerne, Switzerland). From DIV 2 the medium was changed to the differentiation medium containing Neurobasal medium, B-27 ${ }^{\circledR}$ Supplement (50X), N-2 Supplement (100X), $0.6 \mathrm{mM}$ glutamine and penicillin/streptomycin (all Invitrogen Inc., Lucerne, Switzerland).

\section{ELECTROPHYSIOLOGY}

Cover slips with neurons were transferred to a bath chamber mounted to an inverted microscope (Axiovert 25, Carl Zeiss $\mathrm{GmbH}$, München, Germany). Experiments were performed on DIV 0-8 and DIV 11-23 neurons in culture using the whole-cell voltage-clamp technique. Data were collected using a Multiclamp 700A amplifier (Axon Instruments, Union City, CA, USA). We used electrodes with an open tip resistance of 4-5 $\mathrm{M} \Omega$ obtained by pulling borosilicate pipettes (Clark, Warner Instruments Inc., Edenbridge, United Kingdom) with $1.5 \mathrm{~mm}$ external diameter and $1.17 \mathrm{~mm}$ internal diameter without filament to a tip diameter of $\sim 1 \mu \mathrm{m}$ on a horizontal Puller (DMZ Puller, Zeitz GmbH, Martinsried, Germany). The intracellular solution was adapted to the medium the cells were cultivated in; for N2 medium it contained (mM): $110 \mathrm{~K}$-D-gluconate, $5 \mathrm{KCl}, 11$ Tris-phosphocreatine, 1 EGTA, 4.5 MgATP, 10 HEPES, 0.3 Tris-GTP (pH 7.4 with KOH, $290 \mathrm{mOsm}$ ). The extracellular solution for cells coming from N2 medium used for DIV 0 and DIV 1 contained (in mM): $120 \mathrm{NaCl}$, $29 \mathrm{NaHCO}_{3}, 4 \mathrm{KCl}, 1 \mathrm{CaCl}_{2}, 0.7 \mathrm{MgCl}_{2}, 18$ glucose, $\mathrm{pH} 7.4$ when bubbled continuously with $95 \% \mathrm{O}_{2}$ and $5 \% \mathrm{CO}_{2}$. Intracellular solution for cultures coming from complete medium contained (mM): $100 \mathrm{~K}-\mathrm{D}$-gluconate, $5 \mathrm{NaCl}, 1$ EGTA, 5 MgATP, 10 HEPES, and 0.5 Tris-GTP (pH 7.4 with $\mathrm{KOH}, 210$ mOsm). The extracellular solution for complete medium contained (in $\mathrm{mM}$ ): $125 \mathrm{NaCl}$, $26 \mathrm{NaHCO}_{3}, 1.25 \mathrm{NaH}_{2} \mathrm{PO}_{4}{ }^{\star} \mathrm{H}_{2} \mathrm{O}, 2.5 \mathrm{KCl}, 1.0 \mathrm{MgSO}_{4}, 2.0 \mathrm{CaCl}_{2}$ and 11 glucose, pH 7.4 when bubbled continuously with $95 \% \mathrm{O}_{2}$ and $5 \% \mathrm{CO}_{2}$. Voltage-gated sodium- and potassium channels were detected in voltage-clamp mode at a holding potential of $-60 \mathrm{mV}$. The holding potential was changed in a stepwise fashion from -75 to $+25 \mathrm{mV}$ in $5 \mathrm{mV}$ increments for $800 \mathrm{~ms}$ and the voltagegated peak inward current and the sustained outward current (between 600-800 ms) were measured for each step. The inward currents were tetrodotoxin (TTX) sensitive, while the outward currents were blocked by tetraethyl-ammonium and 4-aminopyridine [TEA (3 mM) and 4-AP (1 mM; data not shown)]. For statistical comparisons the maximal evoked currents for a given cell were used. RMPs and APs were recorded in current-clamp mode. Somatic current injections were applied in $2.5 \mathrm{pA}$ steps from -2.5 to $+30 \mathrm{pA}$, in older cells up to $+60 \mathrm{pA}$. Synaptic activity was measured in voltage-clamp mode using the same K-gluconate based internal in order to maximise cell-yield: to detect spontaneous excitatory synaptic currents (sEPSCs), cells were held at $-60 \mathrm{mV}$, while for spontaneous inhibitory synaptic currents (sIPSCs), cells were held at $-40 \mathrm{mV}$. This allowed us to unambiguously identify EPSCs and IPSCs in the same recording without pharmacological agents present, which might have affected network activity. Responses were filtered at $5 \mathrm{kHz}$ and digitized at $20 \mathrm{kHz}$. The excitatory glutamate receptor blocker 2,3-dihydroxy-6-nitro7-sulfamoyl-benzo[f] quinoxaline-2,3-dione (NBQX; $10 \mu \mathrm{M}$ ) and antagonists of inhibitory $\mathrm{GABA}_{\mathrm{A}}$ receptors picrotoxin $(100 \mu \mathrm{M})$ or bicuculline $(20 \mu \mathrm{M})$ were added to the perfusate at the end of recordings to block the respective synaptic activity (Tocris Inc., Bristol, United Kingdom). All other chemicals were purchased from the same supplier (Sigma Inc., Buchs, Switzerland). Recorded sEPSC and sIPSC were detected and analyzed using Mini Analysis 6 (Synaptosoft, Decatur, Georgia). All other data analysis was done with IGOR PRO 6.0 (Wavemetrics, Lake Oswego, OR, USA) software. Two-way ANOVA were used for all statistical analysis (unless otherwise mentioned), with Tukey's HSD tests where indicated.

\section{IMMUNOCYTOCHEMISTRY}

Cells cultured on glass coverslips were rinsed twice with phosphate buffered saline (PBS) pH 7.4 and fixed with 10\% neutral buffered formalin (Sigma Inc., Buchs, Switzerland) for $20 \mathrm{~min}$ at room temperature (RT). After rinsing with PBS, coverslips were permeabilized for $5 \mathrm{~min}$ in $0.2 \%$ TritonX-100/PBS, rinsed with PBS and incubated for $1 \mathrm{~h}$ at RT in a humidified chamber with the following primary antibodies and dilutions (rb: rabbit, ms: mouse): doublecortin (rb, 1:1000, Cell Signaling, Bio Concept, Allschwil, Switzerland), microtubule-associated protein 2 (MAP2; rb, 1:1000, Chemicon, Millipore Inc., Zug, Switzerland), glutamic acid decarboxylase, $67 \mathrm{kDa}$ isoform, (GAD67; ms, 1:500, Chemicon, Millipore Inc., Zug, Switzerland) and synaptophysin (ms, 1:300, Sigma Inc., Buchs, Switzerland). After several washes with PBS, coverslips were incubated for $1 \mathrm{~h}$ with corresponding secondary antibody: Cy5 [donkey anti rabbit IgG 

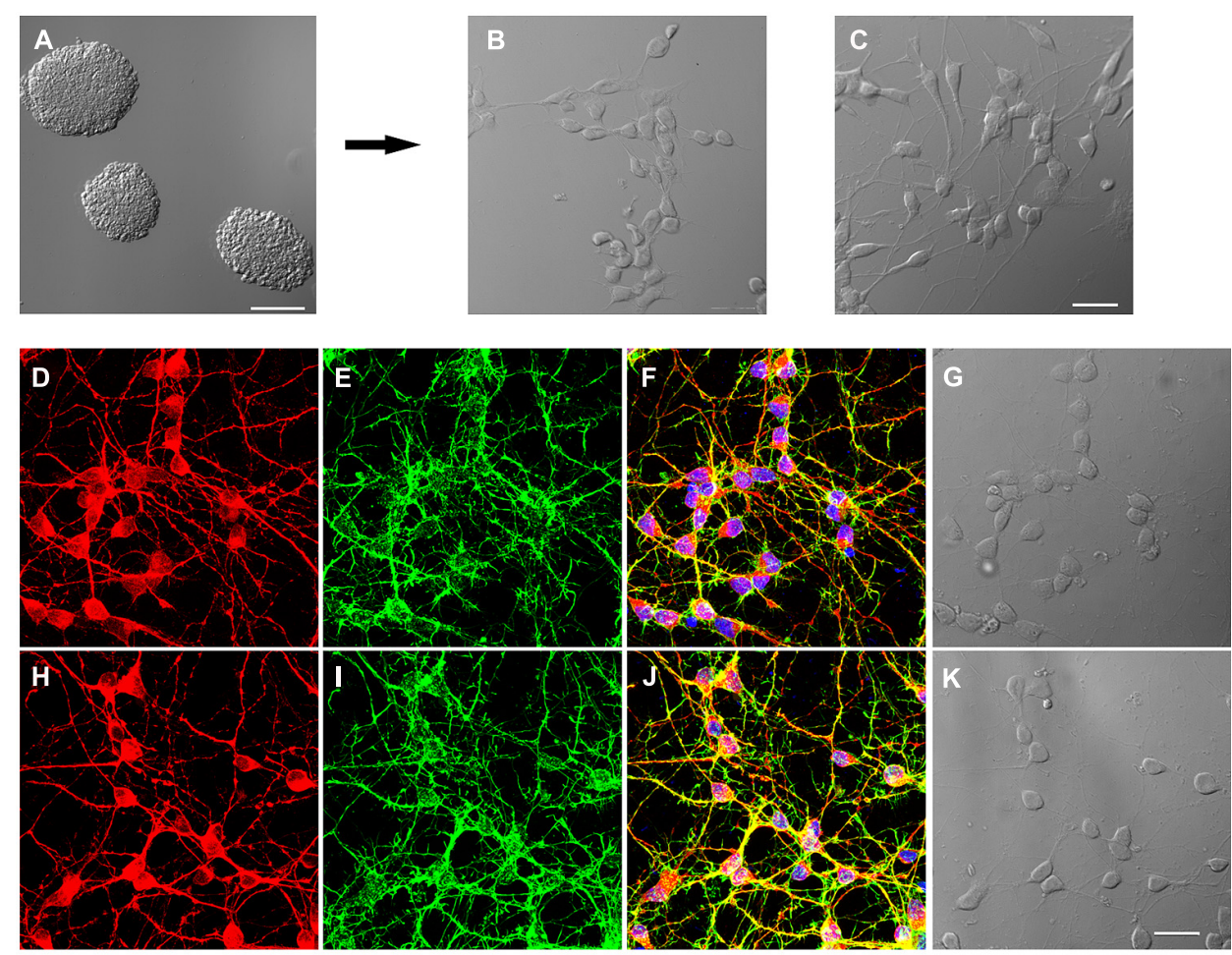

L1

wt

L2

$\mathrm{MeCP} 2-/ \mathrm{y}$
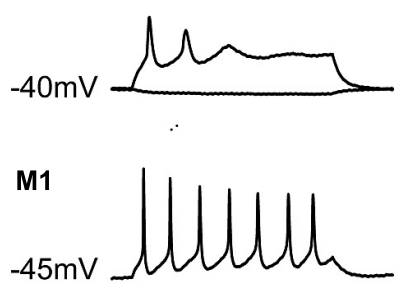

DIV3

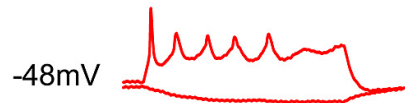

M2

DIV6
FIGURE 1 | mES cell-derived neurons show early maturation.

(A-C) DIC images of early neuronal differentiation in stem cell-derived cultures. (A) Image of embryoid bodies before dissociation; scale bar: $100 \mu \mathrm{m}$. Progenitor cells $6 \mathrm{~h}$ after plating (B) and at DIV 1 (C); scale bar: $20 \mu \mathrm{m}$. Immunostainings against doublecortin (D), actin staining with phalloidin (E). (F) Overlay of doublecortin and phalloidin stain with DAPI stain (blue) and the corresponding DIC image (G) in wt cultures
(H+L), 1:300, Immuno Jackson, Suffolk, United Kingdom], Alexa 488 [donkey anti mouse IgG $(\mathrm{H}+\mathrm{L}), 1: 800$, Invitrogen Inc., Lucerne, Switzerland], Alexa 488-phalloidin (1:400, Molecular Probes, Eugene, OR, USA) and 4',6-diamidino-2-phenylindole (DAPI) (1:1000, Molecular Probes, Eugene, OR, USA). After several washes in PBS, coverslips were mounted in Mowiol1188 as previously described (Baschong et al., 1999). Confocal sections were recorded with a confocal laser scanning microscope Leica TCS SPE with DMI 4000B (Leica Switzerland) and processed with Imaris software (Bitplane, Zurich, Switzerland) and Adobe Photoshop version 10.0 (Adobe Inc., San Jose, CA, USA).

\section{RESULTS}

\section{MORPHOLOGICAL DEVELOPMENT}

We differentiated MeCP2+/y (wt) and MeCP2-/y mES cells from the same E14Tg2a background into neurons using an established protocol (Bibel et al., 2007). Progenitors dissociated from embryoid bodies (Figure 1A) showed already at DIV 0 a distinct, spindle shaped morphology (Figure 1B), which transformed into a multipolar shape by DIV 1 (Figure 1C). To characterize their morphological development, wt (Figures 1D-F) and MeCP2-/y cells (Figures $\mathbf{1 H}-\mathbf{J}$ ) were stained on DIV 3 with antibodies against doublecortin (Figures 1D,H), a marker of immature neurons. In addition we used the actin cytoskeleton label phalloidin 
(Figures 1E,I), the nuclear marker DAPI (Figures 1F,J) and differential interference contrast (DIC) images (Figures 1G,K). By DIV 3 more than $90 \%$ of the cells in culture were immature neurons with a multipolar shape. The DAPI staining indicated that the cell densities were similar in both genotypes (wt: $21.4 \pm 2.2$ cells $/ 10000 \mu \mathrm{m}^{2}, n=10$; MeCP2-/y: $21.8 \pm 3.0$ cells $/ 10000 \mu \mathrm{m}^{2}$, $n=10, p>0.91$ Student's $t$-test).

\section{FUNCTIONAL DEVELOPMENT OF VOLTAGE-GATED CURRENTS}

The wt progenitors exhibited a RMP of $-56 \pm 2.2 \mathrm{mV}$ at DIV $0(n=15)$ compared to $-35 \pm 3.0 \mathrm{mV}$ for MeCP2-/y progenitors $(n=8)$. At DIV 2 RMP of wt cells $(n=21)$ depolarized to $-41 \pm 3.3 \mathrm{mV}$, while for MeCP2-/y $(n=16)$ we measured a RMP of $-36 \pm 2.1 \mathrm{mV}$. In the following days cells from both genotypes showed a gradual hyperpolarization to $-57 \pm 0.9 \mathrm{mV}$ for wt and $-57 \pm 1.0 \mathrm{mV}$ for MeCP2-/y, which stabilized around DIV 11 ( $n=180$ for wt and 151 for MeCP2-/y). Statistical analysis revealed a highly significant influence of developmental age $(p<0.01)$, but no significant effect of the genotype $(p>0.08)$ on the RMP.

We observed voltage-dependent inward sodium currents $\left(\mathrm{I}_{\mathrm{Na}}\right)$ with fast activation and inactivation kinetics, as well as slow, noninactivating outward potassium currents $\left(\mathrm{I}_{\mathrm{K}}\right)$ during all stages of differentiation. Already $6 \mathrm{~h}$ after plating in 6 of 10 cells a small $\mathrm{I}_{\mathrm{Na}}$ of $0.04 \pm 0.02 \mathrm{nA}$ in wt and $0.02 \pm 0.01 \mathrm{nA}$ in MeCP2-/y and a $\mathrm{I}_{\mathrm{K}}$ of $0.11 \pm 0.03 \mathrm{nA}$ in wt and $0.04 \pm 0.01 \mathrm{nA}$ in MeCP2-/y could be found. Both types of currents showed a substantial increase during development in culture (Figure 2). $\mathrm{I}_{\mathrm{Na}}$ increased continuously from $0.48 \pm 0.04 \mathrm{nA}$ in wt and $0.60 \pm 0.04 \mathrm{nA}$ in MeCP2-/y (DIV $3-5$ ) to $3.06 \pm 0.16 \mathrm{nA}$ in wt and $3.14 \pm 0.23 \mathrm{nA}$ in MeCP2-/y (DIV 20-23; Figure 2E). For $\mathrm{I}_{\mathrm{K}}$ an initial increase from $0.36 \pm 0.025 \mathrm{nA}$ in wt and $0.45 \pm 0.03 \mathrm{nA}$ in MeCP2-/y (DIV 3-5) to $1.24 \pm 0.07 \mathrm{nA}$ in wt and $1.78 \pm 0.11 \mathrm{nA}$ in MeCP2-/y (DIV 11-14) then leveled off at $1.52 \pm 0.09 \mathrm{nA}$ in wt and $1.31 \pm 0.09 \mathrm{nA}$ in MeCP2-/y (DIV 20-23; Figure 2F). For $\mathrm{I}_{\mathrm{Na}}$ the effect of genotype was not significant $(p>0.82)$, while the developmental age exerted a highly significant effect ( $p<0.01 ; n=25$ per day and genotype). Both genotype and developmental age significantly affected $\mathrm{I}_{\mathrm{K}}(p<0.01$ for both variables, $n=25$ per day and genotype). Pairwise post hoc analysis did not reveal a significant difference between genotypes at any specific time point $(p>0.5)$.

\section{ACTION POTENTIALS AND EXCITABILITY}

Action potentials were detected from DIV 3 (Figure 1L) in both genotypes. Typical APs are shown for DIV 6 (Figure 3A), DIV 12 (Figure 3B) and DIV 21 (Figure 3C). A quantitative analysis of the APs was performed between the age ranges DIV 4-6, DIV 11-13, and DIV 20-23 in $61 \mathrm{wt}$ and $44 \mathrm{MeCP} 2-/ \mathrm{y}$ cells (Figures 3D-G) by measuring the size of the first AP in a train (see Figure 1M) and its half-width, as well as the initial frequency of APs in a train and their frequency adaptation. First AP size (Figure 3D) increased from DIV 4 to DIV 23 in wt neurons from $59.5 \pm 2.5$ to $87.6 \pm 3.7 \mathrm{mV}$ and in MeCP2-/y from $60.3 \pm 4.9$ to $95.7 \pm 2.8 \mathrm{mV}$. First AP half width (Figure 3E) decreased over time in wt from $6.7 \pm 0.5$ to $2.2 \pm 0.3 \mathrm{~ms}$ and in MeCP2-/y from $4.4 \pm 0.7$ to $1.6 \pm 0.08 \mathrm{~ms}$. Initial AP frequency within a train (Figure 3F) showed no clear developmental pattern and varied between 10$20 \mathrm{~Hz}$ for both genotypes. Cells from both genotypes exhibited a weak frequency adaptation to between 60 and $90 \%$ of the initial frequency (Figure 3G) throughout their development. Statistical analysis revealed a significant effect of age $(p<0.0 .1)$, but not of genotype ( $p>0.3)$ on AP size. Both age and genotype significantly affected the AP half-width $(p<0.01)$ with a significant interaction $(p<0.01)$. Initial AP frequency was significantly affected by both age $(p<0.01)$ and genotype $(p<0.05)$ without significant interaction $(p>0.2)$. AP frequency adaptation was significantly affected by age $(p<0.05)$, but not by genotype $(p>0.9)$.

\section{SYNAPTIC ACTIVITY}

We detected the first spontaneous excitatory (sEPSCs) and inhibitory (sIPSCs) post-synaptic currents by DIV 11 in neurons from both wt and MeCP2-/y cultures (Figure 4). We measured sEPSCs as inward currents (Figures $4 \mathrm{~A}-\mathrm{C}$ bottom traces) at a holding potential of $-60 \mathrm{mV}$, while sIPSCs were best visible and measured as outward currents at a holding potential of $-40 \mathrm{mV}$ (Figures 4A-C top traces). sEPSCs were blocked by the addition of the AMPA-receptor blocker NBQX $(10 \mu \mathrm{M})$ to the bath medium, whereas sIPSCs were blocked by bath application of the $\mathrm{GABA}_{\mathrm{A}}$ receptor antagonists picrotoxin $(100 \mu \mathrm{M})$ or bicuculline $(20 \mu \mathrm{M})$ respectively, in all cultures tested ( $n=21$; Figure $4 \mathrm{D}$ ).

Between DIV 11-23 the frequency of sEPSCs increased from $26.2 \pm 4.8$ to $71.8 \pm 7.4$ events per minute in wt $(n=186)$ and from $21.2 \pm 2.6$ to $43.8 \pm 4.6$ events per minute in MeCP2-/y $(n=172$; Figure $4 \mathrm{E})$. This represents a highly significant increase with age $(p<0.01)$ as well as a significant reduction at DIV 2023 in excitatory activity in MeCP2-/y cultures compared to wt $(p<0.05)$.

The frequency of sIPSCs rose from $23.5 \pm 5.1$ to $97.8 \pm 13.3$ events per minute between DIV 11-23 for wt neurons, while in neurons from MeCP2-/y cultures it went from $19.7 \pm 3.4$ to $58.8 \pm 7.9$ events per minute over the same time (Figure 4 F). Both developmental age $(p<0.01)$ and the cell's genotypes $(p<0.01)$ exerted a highly significant influence in inhibitory synaptic activity.

To see whether the reduced synaptic activity was accompanied by a change in synaptic density we stained cultures at DIV 12,18 , and 21 with antibodies against the neuronal marker MAP2 and against the presynaptic marker synaptophysin (Figures $5 \mathrm{~A}-\mathrm{F}$ ). We measured synapse density by counting the number of synaptophysin positive puncta along 6-12 MAP2-positive dendrites per age range and genotype. In wt cultures the densities were $61 \pm 2.2$, $89 \pm 8.9$, and $64 \pm 8.1$ puncta/ $100 \mu \mathrm{m}$ at DIV 12,18 , and 21 respectively. In MeCP2-/y cultures the values were $46 \pm 2.6,76 \pm 7.3$, and $81 \pm 7.1$ puncta/100 $\mu \mathrm{m}$ for the same time points. While age had a significant influence on the density of synaptophysin puncta $(p<0.01)$, there was no significant effect of the genotype $(p>0.45)$ on synapse density. We also stained the cultures against GAD67, a specific marker for GABAergic neurons. A clear increase in staining could be observed from DIV 12 to 18 to 21 , without an obvious difference in the amount of GAD67 immunoreactivity between the two genotypes (Figures 5G-L). Between DIV 18-21 the percentage of GAD67- and MAP2-double-positive neurons among all MAP2-positive cells was $41+/-3 \%$ for wt and $36+/-2$ 
A1

wt

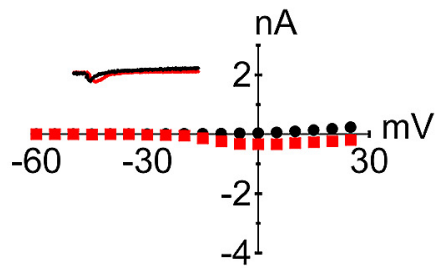

B1

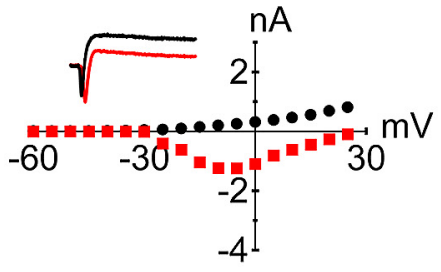

C1

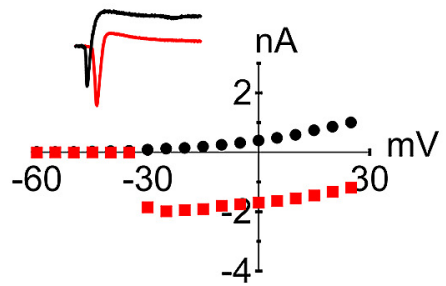

D1

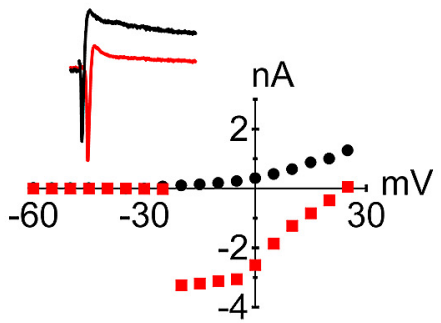

E

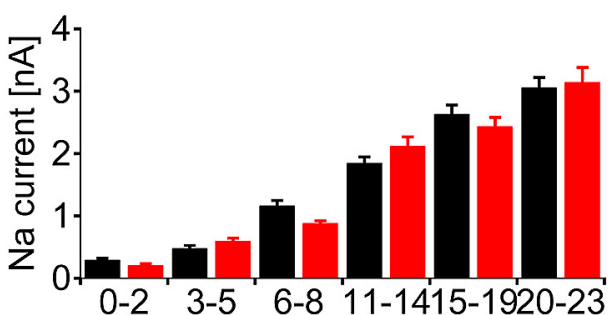

[DIV]

DIV3
A2

MeCP2-/y

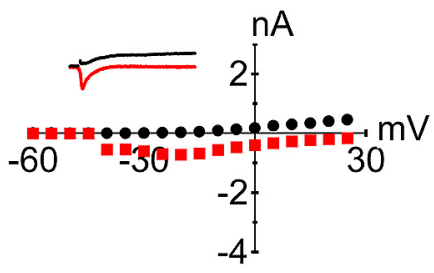

B2

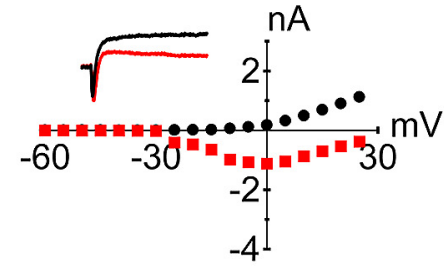

C2

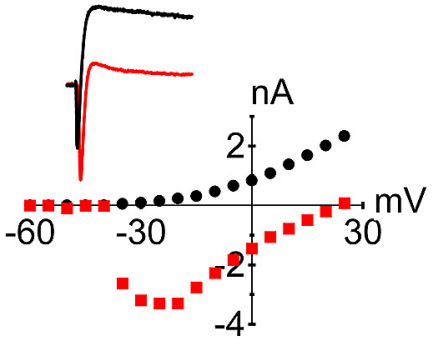

D2

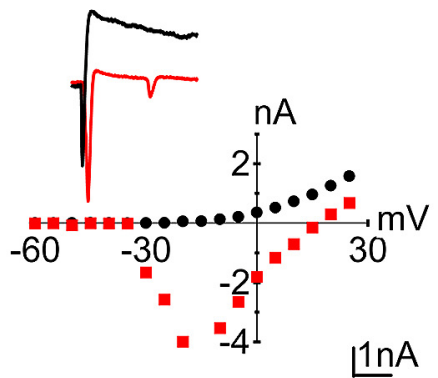

F

$10 \mathrm{~ms}$

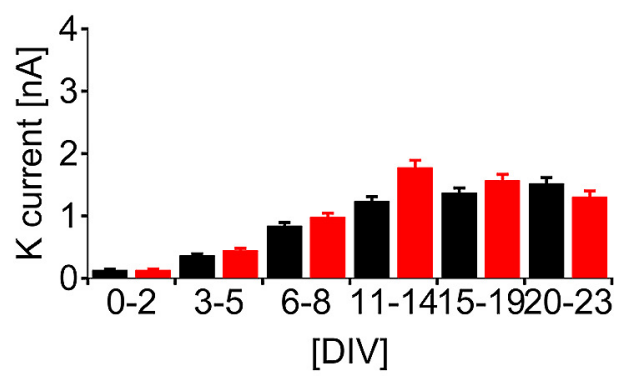

holding potential after depolarization. (A-D) Increase in voltage-activated whole-cell currents from DIV 3 to DIV 21. (E) Comparison of inward sodium current as a function of developmental age for wt (black) and MeCP2-/y (red) cultures. (F) Comparison of outward potassium currents as a function of developmental age for wt (black) and MeCP2-/y (red) cultures. 


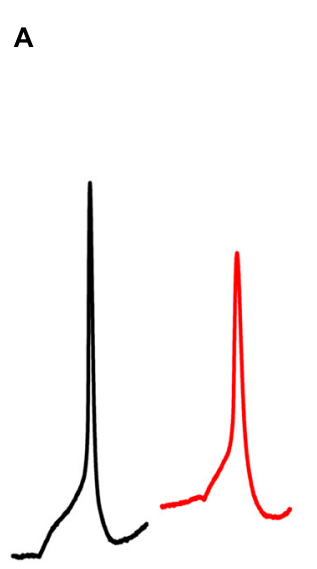

wt MeCP2-/y
B

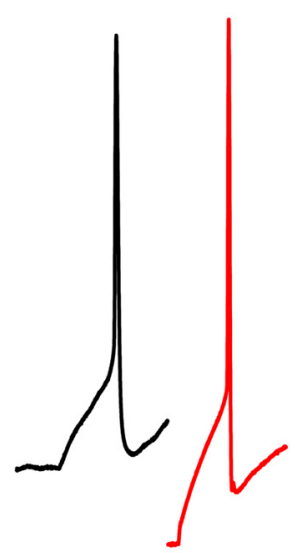

wt MeCP2-/y
C

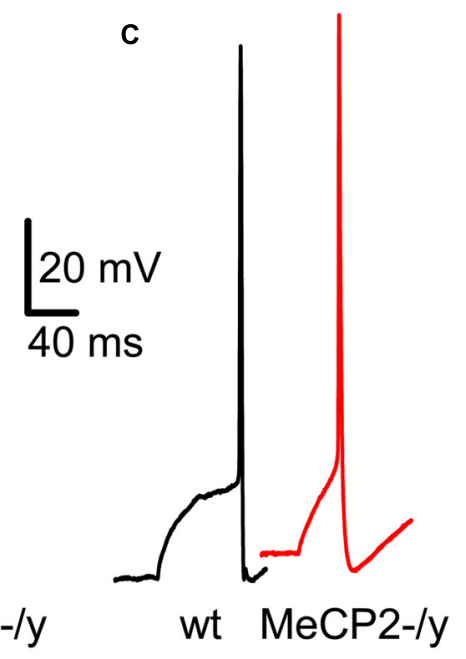

D

E

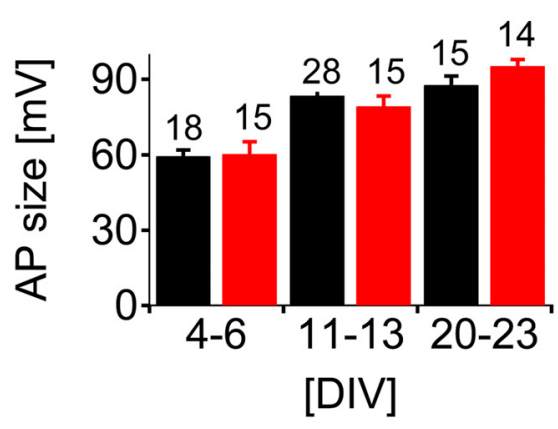

$\mathbf{F}$

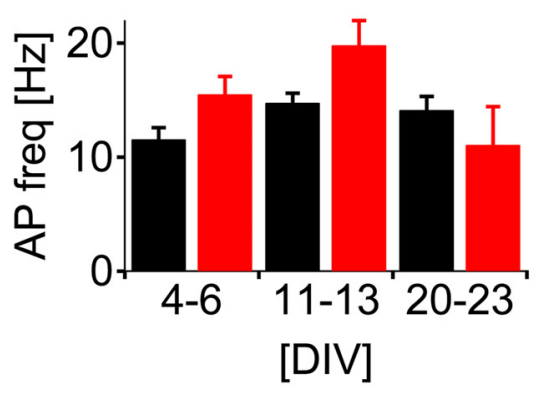

FIGURE 3 | Neuronal spiking patterns as a function of developmental age. Representative single spikes evoked by supra-threshold current injections in cultured neurons at (A) DIV 6, (B) DIV 12, and (C) DIV 21. Repetitive spiking was evoked by current injections shown in Figure 1M. (D-G) Analysis of the spiking

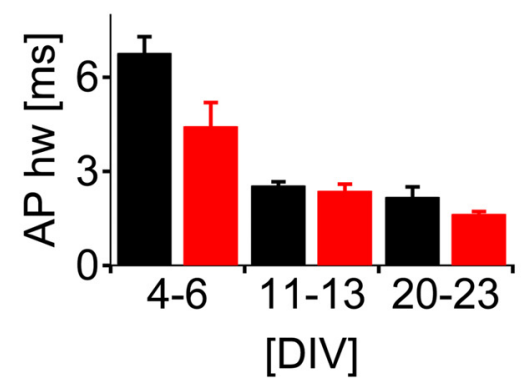

G

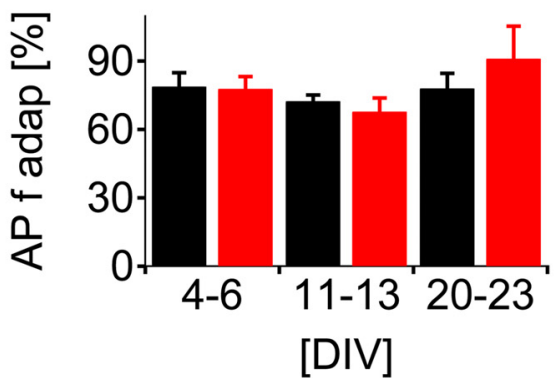

parameters in wt (black) and MeCP2-/y (red) cultures as a function of time in culture. (D) Data for the amplitude of the first $A P$, (E) the half-width at half height for the first $A P$, (F) the initial firing frequency and $(\mathbf{G})$ the frequency at the end of the train relative to the initial firing frequency.
$\%$ for MeCP2 -/y cultures $(n=8$ for each genotype, $p>0.39$, $t$-test).

\section{DISCUSSION}

Networks formed by neurons differentiated from MeCP2-/y mES cells show clear functional deficits compared to wt cultures. Both overall excitatory- and particularly inhibitory synaptic activity was clearly significantly lower in MeCP2-/y compared to wt cultures. Both sEPSCs and sIPSCs appeared at the same age (DIV 11) in MeCP2-/y and wt cultures; and while we could not statistically resolve it, the deficit in synaptic activity appears larger at later compared to early developmental stages. We did not detect a significant difference in the synapse density between wt and MeCP2-/y cultures. A deficit in initial synapse formation or later in the number 
A1

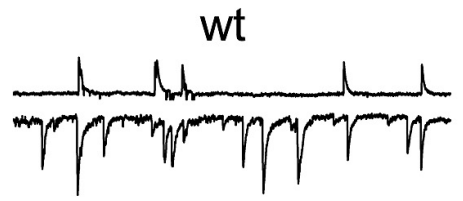

B1
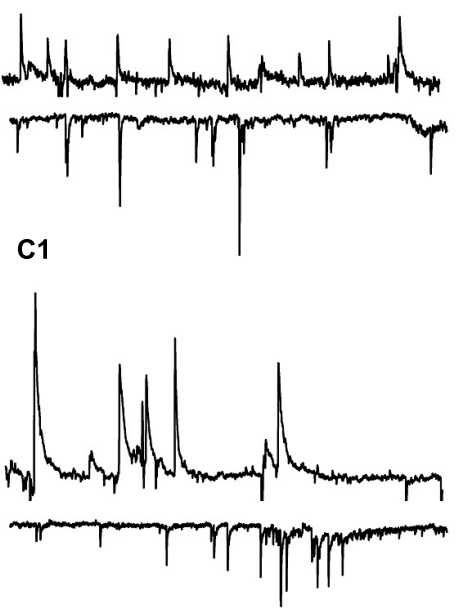

D1

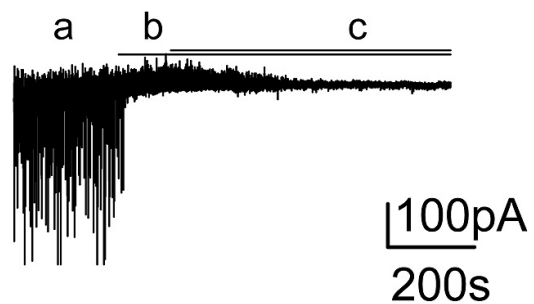

E

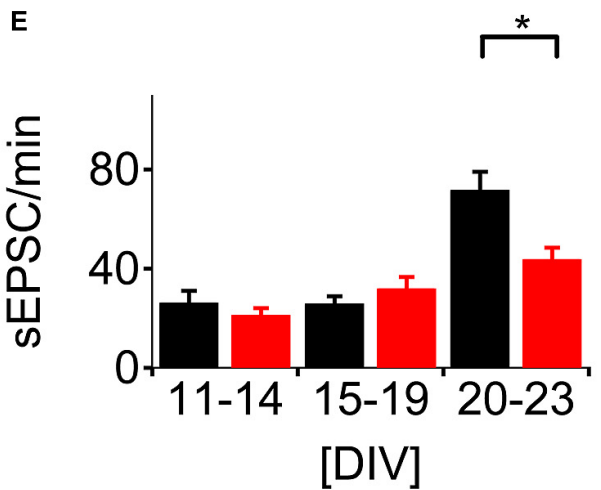

FIGURE 4 | Synaptic activity as a function of development. (A-C) Traces of spontaneous activity in voltage-clamp. Top traces at $-40 \mathrm{mV}$ holding potential bottom traces at $-60 \mathrm{mV}$, respectively. (D) Pharmacological characterization of synaptic currents. (D1) Sample trace of one experiment with baseline recording
A2

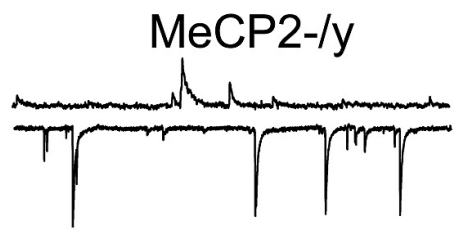

B2

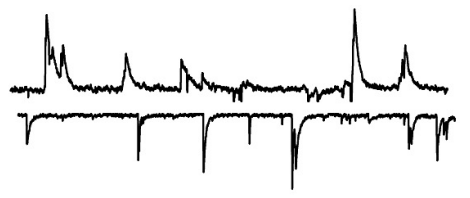

C2

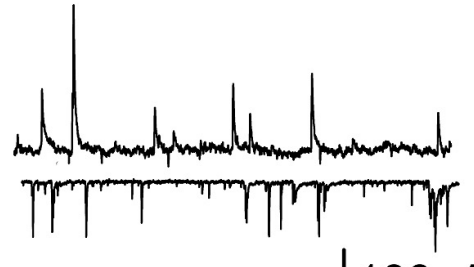

D2

$100 \mathrm{pA}$

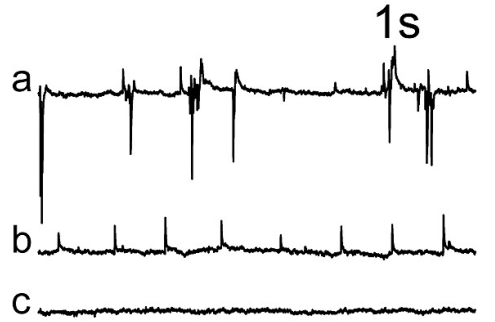

$\mathbf{F}$
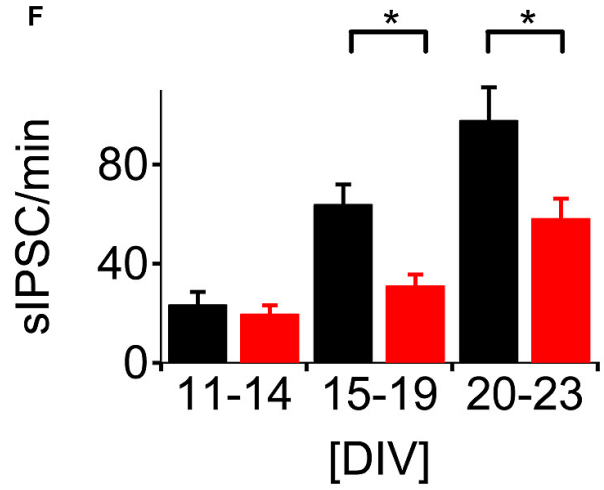

(A), after bath application of NBQX $(10 \mu \mathrm{M})$ and bath application of picrotoxin $(100 \mu \mathrm{M}$; C). (D2) Inserts show the same regions as in (D1) at higher temporal resolution. (E) Frequency of sEPSCs as a function of time in culture for wt (black) and MeCP2-/y (red) cultures. ${ }^{*} p<0.05$. (F) Same data for sIPSCs. ${ }^{*} p<0.01$. 


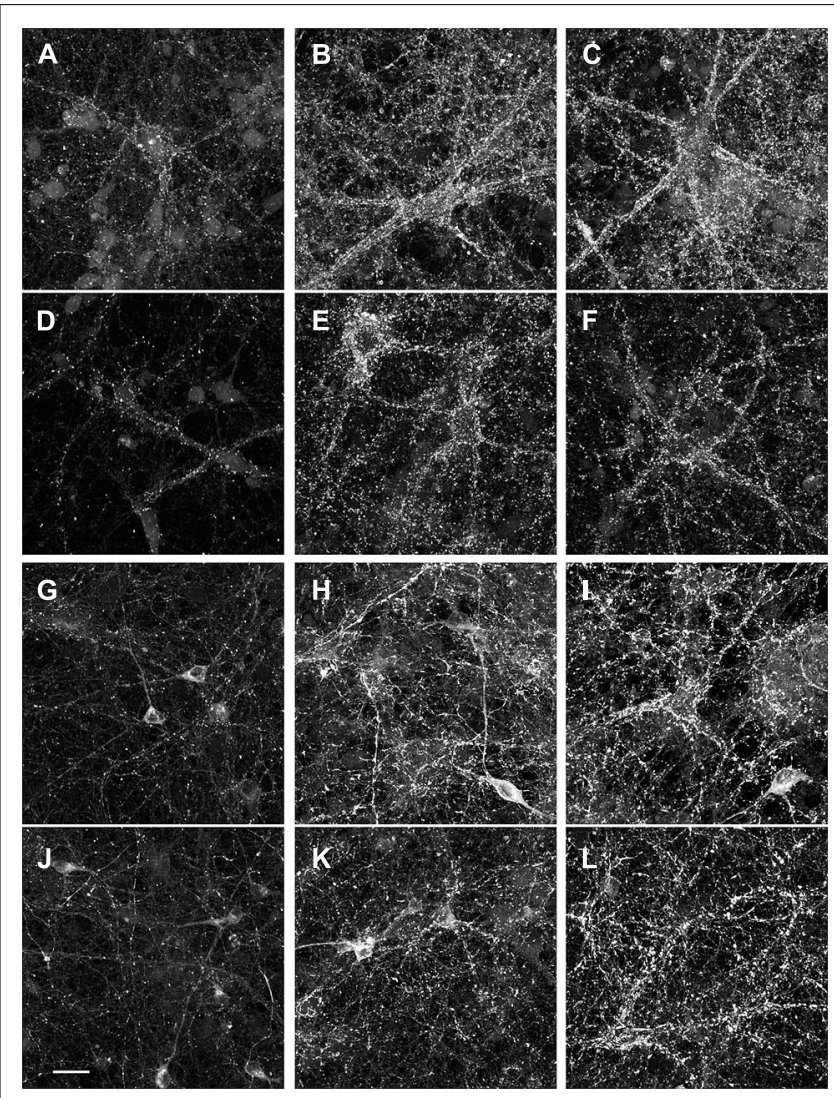

FIGURE 5 | Comparison of synaptic marker expression. Mature neurons from wt (A-C, G-I) and MeCP2-/y (D-F, J-L) were assessed

immunocytochemically on DIV 12, DIV 18 and DIV 21 for expression of the presynaptic marker synaptophysin $(\mathbf{A}-\mathbf{F})$ and the marker of GABAergic neurons GAD-67 (G-L). Scale bar: $20 \mu \mathrm{m}$.

of synapses can therefore not fully explain the reduced synaptic activity. While we did not observe substantial differences in cell densities between MeCP2-/y and wt cultures during development, we cannot rule out subtle differences in cell numbers, which may contribute to our findings. In particular, small changes in the number of interneurons can also not be ruled out as a reason for some of the deficit in GABAergic signaling, even though our GAD67 stains did not show overt differences between the two genotypes. Due to the technical difficulties in unambiguously differentiating between glutamatergic and GABAergic neurons we pooled the recordings from all recorded cells. Since glutamatergic cells are clearly dominant in our cultures, our findings certainly apply to this cell type. For future studies it would be interesting to look at synaptic activity in the different neuron types and to further investigate the mechanisms underlying the deficit in synaptic activity.

We found small, but significant differences in voltage-gated potassium currents and in AP half-with and -frequency between genotypes. The biological significance of these small differences is not clear. Voltage-gated potassium currents are significantly stronger in young MeCP2-/y neurons compared to age-matched wt neurons; this is compatible with the shorter AP half-width that we observe in young $\mathrm{MeCP} 2$ deficient neurons. The AP frequency is significantly higher in MeCP2-/y cultures; however, the effect is not stable over time. Since MeCP2-/y neurons actually show a higher propensity to spike and since the effects on synaptic activity are most pronounced at later developmental stages the lower synaptic activity is clearly not a consequence of lower excitability in MeCP2-/y cultures.

Previous studies using ES and iPS cultures have found differences in the activity of voltage-sensitive sodium channels in cultures of MeCP2-/y neurons (Okabe et al., 2010; Farra et al., 2012). Such differences were not found in another study of cultured neurons (Marchetto et al., 2010). Interestingly we do not find a difference in $\mathrm{I}_{\mathrm{Na}}$, but in $\mathrm{I}_{\mathrm{K}}$ between MeCP2-/y and wt cultures in our system. Several recent studies have looked at the impact of MeCP2 mutations on intrinsic excitability in different brain regions. In one study on locus coeruleus neurons, the authors found altered expression levels of several voltage-dependent conductances in MeCP2-/y mice compared to wt controls (Zhang et al., 2010a). APs had a lower threshold, but were slightly prolonged in MeCP2-/y neurons; the overall effect of the loss of MeCP2 on neuronal excitability in this study depended somewhat on the cell type studied (Zhang et al., 2010a). In other brain regions, such as the cortex, no differences in intrinsic excitability were detected between MeCP2 deficient and wt mice (Dani et al., 2005). MeCP2 expression seems to affect neuronal excitability relatively mildly and in a cell-type dependent manner.

Several groups have studied synapse development and plasticiy in MeCP2 mutated mice (Nelson et al., 2006; Chao et al., 2007; Dani and Nelson, 2009; Wood et al., 2009; Zhang et al., 2010b). In all of these studies, deficits in synaptic maturation either of excitatory, or inhibitory connections were found after a certain developmental delay. This indicates that deficits in connectivity after an initially normal period of synapse formation are a common finding in mouse models of RTT. A lack of fundamental deficits in neuronal excitability, paired with a deficit in synapse maturation is also compatible with RTT patient's symptoms, which occur after a phase of initially normal mental development. We now describe a variant of such a pathology in our mES cell-derived neurons.

The molecular nature of the synaptic deficit has not yet been elucidated for any of the systems tested. The initially normal development and the different effects on either excitatory or inhibitory synaptic transmission in various brain regions argue against key elements of the synaptic release machinery to be affected. In some studies a reduced number of dendritic spines or of synapses was described (Fukuda et al., 2005; Smrt et al., 2007). While we found no difference in the overall density of presynaptic terminals, we cannot be sure that the exact distribution of terminals is the same in MeCP2-/y compared to wt cultures. Different studies have found different types of synapses to be affected by a lack of MeCP2, depending on the brain region studied (Noutel et al., 2011; Durand et al., 2012). Alterations in cell adhesion molecules or scaffolding proteins with differential distributions in different brain regions could explain such findings.

Murine embryonic stem cell cell-derived neuronal cultures can produce relatively homogeneous neuronal tissue with an essentially limitless supply. Transcriptome analysis of such tissue might 
yield candidate molecules responsible for the deficits we observe. Key elements of the known RTT pathology in early developing neural networks could be replicated in our system. This makes it a useful tool to further investigate functional deficits of MeCP2 deficient neurons and to study candidate interventions aimed at slowing or reversing the changes observed.

\section{AUTHOR CONTRIBUTIONS}

Lydia Barth: conception and design, data collection, analysis and interpretation, manuscript preparation. Rosmarie Sütterlin: conception and design, data collection, analysis and interpretation. Markus Nenniger: data analysis and interpretation. Kaspar E. Vogt: conception and design, data analysis and interpretation, manuscript preparation.

\section{ACKNOWLEDGMENT}

We thank Prof. Dr. Yves Barde for providing mES cells.

\section{REFERENCES}

Amir, R. E., Van den Veyver, I. B., Wan, M., Tran, C. Q., Francke, U., and Zoghbi, H. Y. (1999). Rett syndrome is caused by mutations in X-linked MECP2, encoding methyl-CpG-binding protein 2. Nat. Genet. 23, 185-188. doi: 10.1038/ 13810

Baschong, W., Duerrenberger, M., Mandinova, A., and Suetterlin, R. (1999). Three-dimensional visualization of cytoskeleton by confocal laser scanning microscopy. Methods Enzymol. 307, 173-189. doi: 10.1016/S0076-6879(99) 07013-5

Bibel, M., Richter, J., Lacroix, E., and Barde, Y. A. (2007). Generation of a defined and uniform population of CNS progenitors and neurons from mouse embryonic stem cells. Nat. Protoc. 2, 1034-1043. doi: 10.1038/nprot.2007.147

Chahrour, M., and Zoghbi, H. Y. (2007). The story of Rett syndrome: from clinic to neurobiology. Neuron 56, 422-437. doi: 10.1016/j.neuron.2007.10.001

Chang, Q., Khare, G., Dani, V., Nelson, S., and Jaenisch, R., (2006). The disease progression of Mecp2 mutant mice is affected by the level of BDNF expression. Neuron 49, 341-348. doi: 10.1016/j.neuron.2005.12.027

Chao, H. T., Zoghbi, H. Y., and Rosenmund, C. (2007). MeCP2 controls excitatory synaptic strength by regulating glutamatergic synapse number. Neuron $56,58-65$. doi: 10.1016/j.neuron.2007.08.018

Chao, H.-T., Chen, H., Samaco, R. C., Xue, M., Chahrour, M., Yoo, J., et al. (2010). GABAergic dysfunction mediates autism-like stereotypies and Rett syndrome phenotypes. Nature 468, 263-269. doi: 10.1038/nature09582

Chen, R. Z., Akbarian, S., Tudor, M., and Jaenisch, R. (2001). Deficiency of methylCpG binding protein-2 in CNS neurons results in a Rett-like phenotype in mice. Nat. Genet. 27, 327-331. doi: 10.1038/85906

Colic, S., Wither, R. G., Zhang, L., Eubanks, J. H., and Bardakjian, B. L. (2013). Characterization of seizure-like events recorded in vivo in a mouse model of Rett syndrome. Neural Netw. 46, 109-115. doi: 10.1016/j.neunet.2013. 05.002

Dani, V. S., and Nelson, S. B. (2009). Intact long-term potentiation but reduced connectivity between neocortical layer 5 pyramidal neurons in a mouse model of Rett syndrome. J. Neurosci. 29, 11263-11270. doi: 10.1523/JNEUROSCI.101909.2009

Dani, V. S., Chang, Q., Maffei, A., Turrigiano, G. G., Jaenisch, R., and Nelson, S. B. (2005). Reduced cortical activity due to a shift in the balance between excitation and inhibition in a mouse model of Rett syndrome. Proc. Natl. Acad. Sci. U.S.A. 102, 12560-12565. doi: 10.1073/pnas.0506071102

Durand, S., Patrizi, A., Quast, K. B., Hachigian, L., Pavlyuk, R., Saxena, A., etal. (2012). NMDA receptor regulation prevents regression of visual cortical function in the absence of Mecp2. Neuron 76, 1078-1090. doi: 10.1016/j.neuron.2012.12.004

Farra, N., Zhang, W. B., Pasceri, P., Eubanks, J. H., Salter, M. W., and Ellis, J. (2012). Rett syndrome induced pluripotent stem cell-derived neurons reveal novel neurophysiological alterations. Mol. Psychiatry 17, 1261-1271. doi: $10.1038 / \mathrm{mp} .2011 .180$
Fukuda, T., Itoh, M., Ichikawa, T., Washiyama, K., and Goto, Y. (2005). Delayed maturation of neuronal architecture and synaptogenesis in cerebral cortex of Mecp2-deficient mice. J. Neuropathol. Exp. Neurol. 64, 537-544.

Guy, J., Gan, J., Selfridge, J., Cobb, S., and Bird, A. (2007). Reversal of neurological defects in a mouse model of Rett syndrome. Science 315, 1143-1147. doi: $10.1126 /$ science. 1138389

Guy, J., Hendrich, B., Holmes, M., Martin, J. E., and Bird, A. (2001). A mouse Mecp2-null mutation causes neurological symptoms that mimic Rett syndrome. Nat. Genet. 27, 322-326. doi: 10.1038/85899

Hagberg, B., and Hagberg, G. (1997). Rett syndrome: epidemiology and geographical variability. Eur. Child Adolesc. Psychiatry 6(Suppl. 1), 5-7.

Huang, Z. J., Di Cristo, G., and Ango, F. (2007). Development of GABA innervation in the cerebral and cerebellar cortices. Nat. Rev. Neurosci. 8, 673-686. doi: 10.1038/nrn2188

Marchetto, M. C., Carromeu, C., Acab, A., Yu, D., Yeo, G. W., Mu, Y., et al. (2010). A model for neural development and treatment of Rett syndrome using human induced pluripotent stem cells. Cell 143, 527-539. doi: 10.1016/j.cell.2010. 10.016

Moretti, P., and Zoghbi, H. Y. (2006). MeCP2 dysfunction in Rett syndrome and related disorders. Curr. Opin. Genet. Dev. 16, 276-281. doi: 10.1016/j.gde.2006.04.009

Nelson, E. D., Kavalali, E. T., and Monteggia, L. M. (2006). MeCP2-dependent transcriptional repression regulates excitatory neurotransmission. Curr. Biol. 16, 710-716. doi: 10.1016/j.cub.2006.02.062

Nissenkorn, A., Gak, E., Vecsler, M., Reznik, H., Menascu, S., and Ben Zeev, B. (2010). Epilepsy in Rett syndrome - the experience of a National Rett Center. Epilepsia 51, 1252-1258. doi: 10.1111/j.1528-1167.2010. 02597.x

Noutel, J., Hong, Y. K., Leu, B., Kang, E., and Chen, C. (2011). Experience-dependent retinogeniculate synapse remodeling is abnormal in MeCP2-deficient mice. Neuron 70, 35-42. doi: 10.1016/j.neuron.2011. 03.001

Okabe, Y., Kusaga, A., Takahashi, T., Mitsumasu, C., Murai, Y., Tanaka, E., et al. (2010). Neural development of methyl-CpG-binding protein 2 null embryonic stem cells: a system for studying Rett syndrome. Brain Res. 1360, 17-27. doi: 10.1016/j.brainres.2010.08.090

Rett, A. (1966). [On a unusual brain atrophy syndrome in hyperammonemia in childhood]. Wien. Med. Wochenschr. 116, 723-726.

Shahbazian, M., Young, J., Yuva-Paylor, L., Spencer, C., Antalffy, B., Noebels, J., et al. (2002). Mice with truncated MeCP2 recapitulate many Rett syndrome features and display hyperacetylation of histone H3. Neuron 35, 243-254. doi: 10.1016/S0896-6273(02)00768-7

Smrt, R. D., Eaves-Egenes, J., Barkho, B. Z., Santistevan, N. J., Zhao, C., Aimone, J. B., et al. (2007). Mecp2 deficiency leads to delayed maturation and altered gene expression in hippocampal neurons. Neurobiol. Dis. 27, 77-89. doi: 10.1016/j.nbd.2007.04.005

Wood, L., Gray, N. W., Zhou, Z., Greenberg, M. E., and Shepherd, G. M. (2009). Synaptic circuit abnormalities of motor-frontal layer $2 / 3$ pyramidal neurons in an RNA interference model of methyl-CpG-binding protein 2 deficiency. J. Neurosci. 29, 12440-12448. doi: 10.1523/JNEUROSCI.332109.2009

Zhang, X., Cui, N., Wu, Z., Su, J., Tadepalli, J. S., Sekizar, S., et al. (2010a). Intrinsic membrane properties of locus coeruleus neurons in Mecp2-null mice. Am. J. Physiol. Cell Physiol. 298, C635-C646. doi: 10.1152/ajpcell.004 42.2009

Zhang, Z. W., Zak, J. D., and Liu, H. (2010b). MeCP2 is required for normal development of GABAergic circuits in the thalamus. J. Neurophysiol. 103, 24702481. doi: 10.1152/jn.00601.2009

Zhou, Z., Hong, E. J., Cohen, S., Zhao, W. N., Ho, H. Y., Schmidt, L., et al. (2006). Brain-specific phosphorylation of MeCP2 regulates activity-dependent Bdnf transcription, dendritic growth, and spine maturation. Neuron 52, 255-269. doi: 10.1016/j.neuron.2006.09.037

Zoghbi, H. Y. (2003). Postnatal neurodevelopmental disorders: meeting at the synapse? Science 302, 826-830. doi: 10.1126/science.1089071

Conflict of Interest Statement: The authors declare that the research was conducted in the absence of any commercial or financial relationships that could be construed as a potential conflict of interest. 
Received: 13 December 2013; accepted: 26 February 2014; published online: 26 March 2014.

Citation: Barth L, Sütterlin R, Nenniger M and Vogt KE (2014) Reduced synaptic activity in neuronal networks derived from embryonic stem cells of murine Rett syndrome model. Front. Cell. Neurosci. 8:79. doi: 10.3389/fncel.2014.00079

This article was submitted to the journal Frontiers in Cellular Neuroscience.
Copyright (c) 2014 Barth, Sütterlin, Nenniger and Vogt. This is an open-access article distributed under the terms of the Creative Commons Attribution License (CC BY). The use, distribution or reproduction in other forums is permitted, provided the original author(s) or licensor are credited and that the original publication in this journal is cited, in accordance with accepted academic practice. No use, distribution or reproduction is permitted which does not comply with these terms. 